\title{
Spontaneous alternation of high and low reactively curious children
}

Forty-eight fifth and sixth grade children were divided into high and low reactively curious groups on the basis of a paper and pencil reactive curiousity scale, and given a series of twenty T-maze tracing tasks. Each group was subdivided into a Constant Stimulus condition and a Varied Stimulus condition. The results showed that high reactively curious children alternated above chance level in both the Constant and Varied conditions, and low reactively curious children alternated about chance level in the Constant condition and above chance level in the Varied condition.

Ellis \& Arnoult (1965) have demonstrated that stimulus novelty is a determinant of spontaneous alternation in nursery school children. In a T-maze tracing task, children alternated significantly above chance if the $T$-maze stimuli remained constant over 20 trials, and significantly below chance if the stimuli varied on each trial. These results would seem to be consistent with Dember and Earl's theory of alternation behavior (1957). Under the condition of nonvarying stimuli the child would be expected to alternate in order to optimize the amount of stimulus change; whereas in the varying condition, the $\mathrm{T}$-maze stimuli were continuously changed and this would appear to provide enough stimulus variation without $S$ alternating from trial to trial.

Penny \& McCann (1961) have developed a reactive curiosity scale purported to measure in part individual differences in the stimulus variation seeking of children in grades four through six. One characteristic of the reactively curious child is that he seeks new, incongruous stimuli and actively varies his stimulation.

The present study determined the relationship between fifth and sixth grade children's reactive curiosity and their alternation behavior under constant or varied stimulus conditions of the T-maze tracing task used by Ellis and Arnoult.

Method

A total of 98 fifth and sixth grade children were administered the Reactive Curiosity Scale (RCS, Penney \& McCann, 1964) in their classrooms. The RCS is a 90-item self-report test developed by Penney. On the basis of their RCS test scores, 24 Ss scoring highest on the RCS were placed into the High Curious group and 24 lowest scoring $\mathrm{Ss}$ were placed in the Low Curious group. The Ss in each of these groups were then randomly assigned to either the constant or varied stimulus condition for the T-diagram task. This task consisted of drawing lines with felt tip pens onto twenty $\mathrm{T}$-mazes. Each $\mathrm{S}$ was brought into a room individually and was told to draw a line down the T-alley and into one, and only one of the T-arms. When the $S$ entered the room all twenty $\mathrm{T}$-diagrams were laid out on a table but covered with a $9 \times 12$ piece of white construction paper. After $S$ drew a line down the alley and into one of the arms, the T-diagram was removed and another was placed in front of him.

The $T$-diagrams for the constant stimulus condition consisted of black T-outlines on a white background and were marked by black felt tip pens. For the varied stimulus condition a different marker pen, T-outline, background color combination was used for each trial. The T-diagrams were 4-1/2 in. in height and width and were drawn on sheets of $4-1 / 2 \times 6$ in. construction paper. The width of the diagram was 1 in. in both vertical and the horizontal arms.

\section{Results}

Table I shows the percent of Ss alternating on each trial. A $x^{2}$ analysis was applied to the number of alternations for each group to determine the probability of chance occurrence. The analysis revealed that the High RC Ss alternated significantly above chance on both Constant and Varied conditions (Constant: $\mathrm{X}^{2}=4.86$; $1 \mathrm{df} ; \mathrm{p}<.05$. Varied: $\mathrm{x}^{2}=10.74 ; 1 \mathrm{df} ; \mathrm{p}<.01$ ). Low RC Ss alternated about chance level on Constant condition $\left(\mathrm{X}^{2}=2.81 ; 1 \mathrm{df} ; \mathrm{p}<.10\right)$, but significantly above chance on the Varied condition $\left(X^{2}=17.32 ; 1\right.$ df; $\left.p<.01\right)$.

The difference between the number of alternations in

Table 1. Percentage Alternation

\begin{tabular}{|c|c|c|c|c|}
\hline \multicolumn{3}{|c|}{$\mathrm{HIGH}$ RC Ss } & \multicolumn{2}{|c|}{ LOW RC Ss } \\
\hline Trial & Constant & Varied & Constant & Varied \\
\hline 1 & 54 & 82 & 40 & 50 \\
\hline 2 & 69 & 55 & 40 & 79 \\
\hline 3 & 69 & 73 & 40 & 50 \\
\hline 4 & 54 & 73 & 20 & 57 \\
\hline 5 & 54 & 73 & 40 & 79 \\
\hline 6 & 54 & 64 & 40 & 93 \\
\hline 7 & 62 & 55 & 50 & 50 \\
\hline 8 & 62 & 73 & 60 & 64 \\
\hline 9 & 69 & 64 & 60 & 71 \\
\hline 10 & 77 & 36 & 40 & 71 \\
\hline 11 & 69 & 36 & 40 & 57 \\
\hline 12 & 46 & 82 & 50 & 79 \\
\hline 13 & 54 & 73 & 30 & 43 \\
\hline 14 & 77 & 73 & 50 & 86 \\
\hline 15 & 69 & 82 & 30 & 64 \\
\hline 16 & 54 & 55 & 70 & 79 \\
\hline 17 & 46 & 73 & 40 & 71 \\
\hline 18 & 46 & 82 & 20 & 79 \\
\hline 19 & 62 & 73 & 40 & 64 \\
\hline Mean & 60.32 & 66.99 & 42.11 & 67.67 \\
\hline
\end{tabular}


the Constant and Varied conditions was subjected to an F test for both High and Low RC Ss. The analysis showed that the Low RC Ss alternated significantly more in the Varied condition than in the Constant condition $(F=4.96 ; 1.22 \mathrm{df} ; \mathrm{p}<.05)$. The number of alternations for the High RC Ss was also larger in the Varied condition than in the Constant condition, but this difference was not significant $(\mathrm{F}<1)$.

\section{Discussion}

Under the condition of constant stimuli, it was predicted that High RC Ss would alternate above chance occurrence when tracing a line on the T-diagram, whereas the Low RC Ss would alternate slightly below chance. The results support these predictions and indicate that the RCS measures some attribute of a child which is later manifested on an alternation task. If, as Dember and Earl have suggested, alternation behavior is but one manifestation of a tendency of organisms to optimize the amount of stimulus change, then it seems reasonable to assume that what the RCS is, in fact, partially measuring is the amount of stimulus variation that is optimal for each child.

The results under the varied stimulus conditions are less clearcut. It had been expected, on the basis of Ellis and Arnoult's research (1965) that less alternation would be present if the stimuli were varied from trial to trial than if the stimuli remained constant throughout the trials. It also seemed reasonable, using Dember and Earl's theoretical construct, to assume that the varied stimuli would reduce the amount of alternation necessary to maintain an optimal level of stimulus change for any given $S$. The results do not support the predictions.
One possible explanation may lie in the instructions given the Ss before the task was begun. It will be remembered that the $S$ was told he could draw a line to "either side." No mention was made of the possibility of alternation in order to prevent a demand characteristic. It might have been that varying stimuli from trial to trial had the effect of developing a "set" of alternating from trial to trial in the $S$. That is, since the stimuli were different on each trial the $S$ may have taken this difference as a cue that it was permissible, or possibly even mandatory, that he change sides from his previous response. The Ss under the Constant condition, on the other hand, may have developed a "set" of perseveration, since each trial appeared the same as the last.

One difference between the present experiment and that of Ellis and Arnoult, is the age of the Ss. Ellis and Arnoult used nursery school children and the present study used fifth and sixth grade children. At the lower age level more varied stimulation seemed to decrease alternation, while the opposite effect is reported for older children. It might be that varied stimulation had differential effects on alternation behavior at different age levels. This possibility requires systematic study.

\section{References}

Dember, W. N., \& Earl, R. W. Analysis of exploratory, manipulatory, and curiosity behavior. Psychol. Rev., 1957, 64, 91-96.

Ellis, N. C., \& Arnoult, M. D. Novelty as a determinant of spuntaneous alternation in children. Psychon. Sci., 1965, 2, 163-164.

Penney, R. I., \& McCann, B. The children's reactive curiosity scale. Psychol. Rep., 1964, 15, 323-334. 